\title{
Preemptive Fuzzy Goal Programming in Fuzzy Environments
}

\author{
J. Jusufovic, A. Omerovic \\ Faculty of Business Administration \\ Mehmet Can \\ Faculty of Arts and Social Sciences, \\ International University of Sarajevo, Paromlinska 66, \\ 71000 Sarajevo, Bosnia and Herzegovina \\ E-mails: jusufovic@ius.edu.ba amir1608@gmail.com mcan@ius.edu.ba
}

\begin{abstract}
There are a variety of efficient approaches to solve crisp multiple objective decision making problems. However in the real life the input data may not be precisely determined because of the incomplete information. This paper deals with a method which can be applied to solve fuzzy multi objective production marketing problems.
\end{abstract}

\section{INTRODUCTION}

In a standard multiple goal programming, goals and constraints are defined precisely. Fuzzy goal programming has the advantage of allowing for the vague aspirations of decision makers, which are quantified by some natural language rules.

To our knowledge, first R. Narasimhan (1980) introduced fuzzy set theory into objective programming. Since then many achievements have been added to the literature. In the literature there are several approaches for solving fuzzy goal programming, and their application to the production marketing problem. Among these methods there are the ones using preemptive fuzzy goal programming, interpolated membership function, weighted additive model, preference structure on aspiration levels, and nested priority.

\section{MULTIPLE FUZZY GOAL PROGRAMMING}

In a multiple goal programming problem, the optimal realization of multiple objectives is desired under a set of constraints imposed by a real life environment. If the goals and constraints are all expressed with equalities, we have a completely symmetric formulation
Find $\mathbf{x}$

uch that $\mathbf{A x}=\mathbf{b}, \mathbf{x} \geq \mathbf{0}$.

Where $\mathbf{x}$ is the vector of variables, $\mathbf{b}$ is the vector of the goals and available resources, and $\mathbf{A}$ is the matrix of the coefficients. In the cases when the decision maker is not precise in goals and restrictions, the linguistic statements such as "around b" will be used. In this case the above crisp goal programming problem becomes

Find $\mathbf{x}$ such that $\mathbf{A x}=\tilde{\mathbf{b}}, \mathbf{x} \geq \mathbf{0}$.

Where the fuzzy components $b_{i}$ of the fuzzy vector $\mathbf{b}$ can be represented by, for example, triangular fuzzy numbers:

$\mu_{i}(z)=\left\{\begin{array}{c}\left(z-\left(b_{i}-d_{i 1}\right)\right) / d_{i 1}, b_{i}-d_{i 1} \leq z \leq b_{i}, \\ \left(\left(b_{i}+d_{i 2}\right)-z\right) / d_{i 2}, b_{i} \leq z \leq b_{i}+d_{i 2}, \\ 0, \text { elsewhere. }\end{array}\right.$

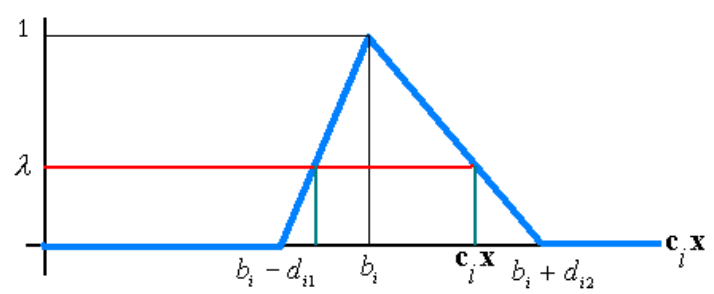

Figure 1. Fuzzy components $b_{i}$ of the fuzzy vector $\mathbf{b}$. 
To have a membership number at least $\lambda$, $\mathbf{C}_{i} \mathbf{X}$ must remain in the interval

$$
b_{i}-d_{i 1}+\lambda d_{i 1} \leq \mathbf{c}_{i} \mathbf{x} \leq b_{i}+d_{i 2}+\lambda d_{i 2}
$$

that is

$$
\left(\mathbf{c}_{i} \mathbf{x}-\left(b_{i}-d_{i 1}\right)\right) / d_{i 1} \geq \lambda,\left(\left(b_{i}+d_{i 2}\right)-\mathbf{c}_{i} \mathbf{x}\right) / d_{i 2} \geq \lambda \text { Find } \mathbf{x} \text {. }
$$

Hence the above fuzzy goal programming problem is the maximum satisfaction problem of the fuzzy equations, and this goal can be achieved by the solution of the below crisp linear programming problem Lai, and Wang (1996).

Max $\lambda$ such that for all $i$, $\left(\mathbf{c}_{i} \mathbf{x}-\left(b_{i}-d_{i 1}\right)\right) / d_{i 1} \geq \lambda$, $\left(\left(b_{i}+d_{i 2}\right)-\mathbf{c}_{i} \mathbf{x}\right) / d_{i 2} \geq \lambda$.

$\lambda \in[0,1]$, and $\mathbf{x} \geq \mathbf{0}$.

In some decision problems, some goals are so important that unless these goals are reached, the decision maker would not consider the achievement of other goals. The method of differentiating goals according their importance is called preemptive fuzzy goal programming

\section{PREEMPTIVE FUZZY GOAL PROGRAMMING}

Let us assume the existence of $\mathbf{K}$ priority levels in the fuzzy goal programming problem (2). The problem is then partitioned in $\mathbf{K}$ sub problems which can be transformed into a standard goal programming problem (6).

The goal set $G_{r}(x)$ has higher priority then the goal set $G_{s}(x)$ if $r<s$. After ordering goals, we will solve the first sub problem by considering the first priority goals $\left(G_{1}(x)\right)$ only:

Find $\mathbf{x}$

Such that

$$
\begin{aligned}
& g_{1 i}(\mathbf{x})=\widetilde{b}_{1 i}, \quad i=1,2, . ., m_{1}, \\
& \mathbf{x} \geq \mathbf{0}, \quad g_{1 i}(\mathbf{x}) \in G_{1}(\mathbf{x})
\end{aligned}
$$

where $m_{1}$ is the number of the goals in the set of first priority goals $\left(G_{1}(x)\right)$. Next the goals in the second priority level $\left(G_{2}(x)\right)$ will be considered; under the condition that achievement of the first sub problem is satisfied:

$$
\begin{gathered}
g_{1 i}(\mathbf{x})=b_{1 i}-d_{1 i}\left(g_{1 i}^{-}-g_{1 i}^{*}\right) \\
i=1,2, . ., m_{1}, \quad \mathbf{x} \geq \mathbf{0}, \quad g_{1 i}(\mathbf{x}) \in G_{1}(\mathbf{x}), \\
g_{2 i}(\mathbf{x})=\tilde{b}_{2 i}, \quad i=1,2, . ., m_{2}, \\
\quad \mathbf{x} \geq \mathbf{0}, \quad g_{2 i}(\mathbf{x}) \in G_{2}(\mathbf{x}),
\end{gathered}
$$

where $g_{1 i}^{-}, g_{1 i}^{*}$ are the maximum and minimum solutions of the first sub problem. Similarly we can solve the third problem under the condition that full achievements of the first and second sub problems are preserved:

Find $\mathbf{x}$ such that

$$
\begin{aligned}
& g_{1 i}(\mathbf{x})=b_{1 i}-d_{1 i}\left(g_{1 i}^{-}-g_{1 i}^{*}\right), \quad i=1,2, . ., m_{1}, \\
& \mathbf{x} \geq \mathbf{0}, \quad g_{1 i}(\mathbf{x}) \in G_{1}(\mathbf{x}), \\
& g_{2 i}(\mathbf{x})=b_{2 i}-d_{2 i}\left(g_{2 i}^{-}-g_{2 i}^{*}\right), \quad i=1,2, . ., m_{2}, \\
& \mathbf{x} \geq \mathbf{0}, \quad g_{2 i}(\mathbf{x}) \in G_{2}(\mathbf{x}),
\end{aligned}
$$

$$
\begin{aligned}
& g_{3 i}(\mathbf{x})=\tilde{b}_{3 i}, \quad i=1,2, . ., m_{3}, \\
& \mathbf{x} \geq \mathbf{0}, \quad g_{3 i}(\mathbf{x}) \in G_{3}(\mathbf{x}),
\end{aligned}
$$

where $g_{2 i}^{-}, g_{2 i}^{*}$ are the maximum and minimum solutions of the second sub problem. This procedure is then repeated until all priority levels are finished.

One can also allow some tolerances in the solution of the sub problems. For example the problem (9) with some tolerances becomes:

Find $\mathbf{x}$

such that

$$
\begin{aligned}
& g_{1 i}(\mathbf{x})=b_{1 i}-d_{1 i}\left(g_{1 i}^{-}-g_{1 i}^{*}\right)-p_{1 i}, \quad i=1,2, . ., m_{1}, \\
& \quad \mathbf{x} \geq \mathbf{0}, \quad g_{1 i}(\mathbf{x}) \in G_{1}(\mathbf{x}),
\end{aligned}
$$




$$
\begin{aligned}
& g_{2 i}(\mathbf{x})=b_{2 i}-d_{2 i}\left(g_{2 i}^{-}-g_{2 i}^{*}\right)-p_{2 i}, \quad i=1,2, . ., m_{2}, \\
& \mathbf{x} \geq \mathbf{0}, \quad g_{2 i}(\mathbf{x}) \in G_{2}(\mathbf{x}), \\
& \quad(10) \\
& g_{3 i}(\mathbf{x})=\widetilde{b}_{3 i}, \quad i=1,2, . ., m_{3}, \\
& \quad \mathbf{x} \geq \mathbf{0}, \quad g_{3 i}(\mathbf{x}) \in G_{3}(\mathbf{x}), \\
& \text { where } p_{1 i}, p_{2 i} \text { are allowable tolerances. }
\end{aligned}
$$

\section{AN APLICATION: THE PRODUCTION- MARKETING PROBLEM}

Assume decision maker consider sale goals only after the profit goal is absolutely achieved. Then the optimization problem is divided into two sub problems. The first problem deals with the first priority goal, profit. Assume profit is computed through the formula

$$
p(x, y)=80 x+40 y
$$

in dollars, when monthly sales are $(x, y)$ items from each kind of products, and the fuzzy goal is:

Find $(x, y)$

such that

$$
p(x, y)=\tilde{b}_{11}, \quad x, y \geq 0 \text {, }
$$

where $\tilde{b}_{1}$ is the fuzzy profit goal with $b_{11}=7000, d_{11}=1000, d_{12}=2000$.

This problem is transformed into the crisp linear programming problem:

Maximize $\lambda$ such that

$$
\begin{aligned}
& p(x, y) \geq 6000+1000 \lambda \\
& p(x, y) \leq 9000-2000 \lambda \\
& 0 \leq \lambda \leq 1, x, y \geq 0 .
\end{aligned}
$$

The solution of the above problem is found to be $x=87.5, y=0, \lambda=1$. For this production plan one has $p(x, y)=7000$ as expected.

Next consider the second priority level of sales

Find $(x, y)$ such that $p(x, y)=7000$,

$$
x=\tilde{b}_{21}, y=\tilde{b}_{22}, x, y \geq 0 .
$$

where $\tilde{b}_{21}$ is the fuzzy sell of the first product with $b_{21}=60, d_{1}=10, d_{2}=20$, and $\tilde{b}_{22}$ is the fuzzy sell of the second product with $b_{22}=40, d_{1}=10, d_{2}=20$.

This problem is transformed into the crisp linear programming problem:

Maximize $\lambda$ such that

$$
\begin{aligned}
& p(x, y)=7000 \\
& x \leq 80-20 \lambda, x \geq 50+10 \lambda, y \leq 60-20 \lambda \\
& y \geq 30+10 \lambda, 0 \leq \lambda \leq 1, x, y \geq 0
\end{aligned}
$$

By the use of the Linear Programming package under MATHEMATICA, the solution of the above problem is found to be $x=65, y=45, \lambda=0.75$.

\section{DISCUSSION}

In this article, to deal with and optimization problem with objectives in two importance levels, the method of preemptive fuzzy goal programming is used. The solution of the first sub problem is found, using this information, the goal in the second priority level is considered; under the condition that achievement of the first sub problem is satisfied. Using $\lambda$-cuts, fuzzy goals are transformed into fuzzy equalities. The obtained crisp linear programming problems are solved by the Linear Programming package under MATHEMATICA. The number of the priority levels can be increased to represent a real world problem without any difficulty.

\section{REFERENCES}

Narasimhan, R., Goal programmimg in a fuzzy environment, Decision Sciences, 11 (1980) 325-338.

Bhattacharya, J.R. Rao, and R.N. Twari, Fuzzy multi-criteria facility location, Fuzzy Sets and Systems, 51 (1992) 277-287.

Lai, Y.J., and Hwang, C.L. Fuzzy Multi Objective Decision Making, Springer, (1996). 